\title{
Individual morbidity and neighbourhood deprivation in a non-metropolitan area
}

\author{
E G Jessop
}

\begin{abstract}
Study objective-The aim was to replicate, in a non-metropolitan area, a study by Curtis based on data from different parts of London which found a significant relationship between individual morbidity and neighbourhood deprivation.

Design-This study used the same design as the previous study. Information on individual morbidity was obtained, using the Nottingham health profile. Deprivation scores were assigned to respondents according the Jarman (and also Townsend) scores of the enumeration district in which they lived. Logistic regression models were built, using the enumeration district as the unit of analysis, to see if, after allowance for age and sex, the Jarman (or Townsend) score significantly improved the prediction of the enumeration district being above or below the sample median to any of the six dimensions of the profile.
\end{abstract}

Setting-The survey involved households in 10 electoral wards in Colchester and Clacton, Essex, United Kingdom, in 1988.

Participants-A systematic sample of 200 persons was drawn from the electoral register in each of 10 wards yielding 2000 names.

Main results-Nottingham health profiles were obtained from 1555 respondents out of an initial sample of 2000 names from the electoral register; the response rate among those alive and resident at a valid address was $93 \%$. Jarman and Townsend scores were assigned to 1496 of the respondents, and the 162 enumeration districts were used as a unit of analysis. Contrary to Curtis's finding, Jarman score did not add significantly in a multiple logistic regression model to the prediction of response to any of the six dimensions of the Nottingham health profile, nor did the Townsend score.

Conclusions-Apart from chance variation, there are two possible explanations for this finding. The measures of deprivation may be valid in London but not elsewhere; or there may be a true difference between cities and towns in the effect that deprivation has on subjective health.

f Epidemiol Community Health 1992; 46: 543-546

Dr Jessop's correspondence address: Wessex Institute Dawn House, Romsey Road Winchester SO22 5DH, United Kingdom

Accepted for publication June 1992

The relationship between deprivation and health is of obvious importance to the practice of public health. The validity of various measures of deprivation, particularly when used for resource allo- cation, has however been debated ${ }^{1-5}$; as have the merits of different subjective measures of health. ${ }^{6}$

In a recent paper, ${ }^{7}$ Curtis was able to demonstrate a relationship between self reported health and neighbourhood deprivation. This study was based in London and used the Nottingham health profile $^{8}$ to measure health, and the Jarman score ${ }^{9}$ (assigned by enumeration district of residence) to characterise deprivation. Whatever the theoretical arguments, therefore, it seems that empirically the Jarman score can, in London at least, be used to predict how people will rate their health. This finding is of considerable importance to health authorities wishing to assess levels of health across an entire resident population but unable to afford comprehensive surveys. Curtis's result, if generalisable, means that census data (which are of course available for the entire resident population in all health districts) can be used as a proxy for health information.

On the other hand it may be that Curtis's finding is only valid in London. There may for example be a difference between metropolitan and other areas in the relationship between social deprivation and health. I have therefore repeated Curtis's methodology, using data from a survey conducted in a non-metropolitan part of south east England. In particular, I have used the same measures of health (the Nottingham health profile) and neighbourhood deprivation (Jarman score assigned by enumeration district). Because of the criticism that the Jarman score is London biased, I have also used Townsend's score ${ }^{5}$ to characterise deprivation.

\section{Methods}

The data examined were collected during a questionnaire survey of 10 electoral wards in the health district of North East Essex during 1988; the survey was designed to examine the relationship between mortality and self rated health at electoral ward level. All electoral wards in North East Essex were ranked by standardised mortality ratio, using all cause all age mortality during the period 1981-1986, and within each decile of the distribution the largest ward was selected for survey. These 10 wards lay within the towns of Colchester and Clacton. (One further ward was included in the survey because of its very low standardised mortality ratio, but results from this small rural ward are not included in the present analysis.)

A sample of 200 names in each ward was tematic sample with a random start point; to this sample was mailed the Nottingham health profile (part I only). Non-responders after one postal 
reminder were visited and the health profile administered by experienced interviewers.

In the present analysis, respondents have been assigned Jarman and Townsend deprivation scores relative to the 640 enumeration districts of North East Essex according to that of their full post code. The survey results were aggregated to enumeration district level, using the mean for age, sex (coded as 1 for males, 2 for females), and each of the six Nottingham health profile dimensions.

Multiple logistic regression was then carried out using SPSS/PC V3.1 to assess whether deprivation scores provided additional explanation, significant at the $5 \%$ level of probability, of response to each of the six dimensions of the Nottingham health profile after inclusion in the equation of mean age and mean sex score for the respondents in the enumeration district as continuous variables. The effect of including a dummy variable for town (Colchester or Clacton) was also explored. Analysis was based on the enumeration district as the unit of analysis because density of sampling meant that there was a median of nine respondents per enumeration district (range 1 to 28), with each respondent in the district being characterised by the same Jarman score. The mean Nottingham health profile scores of the enumeration districts on each dim-

Table I Age and sex of respondents

\begin{tabular}{lllc}
\hline Age (years) & Male & Female & Total \\
\hline $16-44$ & 362 & 351 & 713 \\
$45-64$ & 186 & 216 & 402 \\
65 or more & 172 & 209 & 381 \\
Total & 721 & 776 & 1496 \\
\hline
\end{tabular}

Table II Components of farman score: lower and upper quartile of enumeration district values (equivalent data from Curtis's study in brackets)

\begin{tabular}{|c|c|c|}
\hline \multirow[b]{2}{*}{ Component } & \multicolumn{2}{|c|}{ Quartile $(\%)$} \\
\hline & Lower & Upper \\
\hline Elderly living alone & $2.7(3.5)$ & $7.0 \quad(7.9)$ \\
\hline Under 5 years old & $3 \cdot 8(3 \cdot 1)$ & $8 \cdot 3(6.5)$ \\
\hline One parent household & $0.5(0.7)$ & $3.7 \quad(3.5)$ \\
\hline Unskilled & $0.0(0.0)$ & $5 \cdot 7 \quad(9 \cdot 8)$ \\
\hline Unemployed & $4 \cdot 5(5 \cdot 5)$ & $10.9(12.8)$ \\
\hline Overcrowded & $1.6(3.3)$ & $6 \cdot 2(16 \cdot 4)$ \\
\hline Moved house & $6 \cdot 4(5 \cdot 7)$ & $14 \cdot 6(17 \cdot 1)$ \\
\hline Ethnic & $0.8(4.0)$ & $2.9(13.5)$ \\
\hline
\end{tabular}

Table III Proportion $(\%)$ of respondents reporting problems (nonzero Nottingham health profile score)

\begin{tabular}{lll}
\hline NHP indicator & Present study & Curtis's study \\
\hline Lack of energy & $27 \cdot 4$ & $23 \cdot 3$ \\
Pain & $20 \cdot 5$ & $16 \cdot 1$ \\
Emotional distress & $33 \cdot 0$ & $36 \cdot 0$ \\
Sleep problems & $36 \cdot 4$ & $40 \cdot 0$ \\
Social isolation & $16 \cdot 4$ & $15 \cdot 6$ \\
Physical immobility & $24 \cdot 2$ & $19 \cdot 9$ \\
Base $(=100 \%)$ & 1496 & 1221 \\
\hline
\end{tabular}

Table IV Results of multiple logistic regression

\begin{tabular}{lrclll}
\hline & & Constant & Sex & Age & UPA \\
\hline Energy & Estimate & -3.5235 & 0.8054 & 0.0468 & 0.0200 \\
(median score 13.45) & SEM & 1.5029 & 0.7381 & 0.0164 & 0.0123 \\
Pain & $\mathrm{p}$ & 0.02 & 0.28 & 0.004 & 0.10 \\
(median score 3.76) & Estimate & -3.7619 & 0.2225 & 0.0721 & 0.0062 \\
& $\mathrm{SEM}$ & 1.5589 & 0.7700 & 0.0181 & 0.0126 \\
Emotional distress & $\mathrm{p}$ & 0.02 & 0.77 & 0.0001 & 0.62 \\
(median score 7.60) & Estimate & -1.0417 & 0.4911 & 0.0056 & 0.0073 \\
Sleep problems & SEM & 1.3836 & 0.7003 & 0.0148 & 0.0118 \\
(median score 11.76) & $\mathrm{p}$ & 0.45 & 0.48 & 0.71 & 0.53 \\
& Estimate & -3.9884 & 1.2662 & 0.0417 & 0.0198 \\
Social isolation & SEM & 1.5234 & 0.7458 & 0.0162 & 0.0122 \\
(median score 3.81) & $\mathrm{p}$ & 0.01 & 0.09 & 0.01 & 0.11 \\
& Estimate & 0.0835 & -0.2594 & 0.0077 & -0.0102 \\
Physical immobility & $\mathrm{SEM}$ & 1.3776 & 0.6998 & 0.0149 & 0.0118 \\
(median score 4.48) & $\mathrm{p}$ & 0.95 & 0.71 & 0.60 & 0.39 \\
& Estimate & -3.5316 & -0.1898 & 0.0834 & -0.0115 \\
& $\mathrm{SEM}$ & 1.5879 & 0.7973 & 0.0192 & 0.0130 \\
\hline
\end{tabular}

UPA $=$ Jarman underprivileged area score ension were dichotomised as below (recorded as 0 ) or above (recorded as 1) the median for the sample.

\section{Methods}

In all, 1555 responses were obtained from the initial sample of 2000 names. Of the remainder, 21 had died, 42 were not contactable (either because the address given on the electoral register could not be found, or because on visiting, the named person was not known at the address), 256 had moved away, 43 were in principle contactable but were not contacted after three visits, and 69 refused. Thus the response rate was $78 \%(1555 /$ $2000)$ of the original sample and $93 \%(1555 / 1667)$ of those alive and resident at a valid address.

The present analysis is based on 1496 respondents in 162 enumeration districts; for 56 respondents the post code of the address given on the electoral register could not be ascertained, and for the other three the post code could not be matched to an enumeration district code in the post code computer file. The age and sex of these 1496 respondents are shown in table I.

The mean value of the Jarman score for the 162 enumeration districts was $+3 \cdot 8$, with a range from $-21 \cdot 1$ to $+49 \cdot 0$. The median was +2.9 with quartiles at $-6 \cdot 14$ and $13 \cdot 45$. (Note that all these scores are relative to all enumeration districts in North East Essex.) The parameter values of these enumeration districts at the first and third quartile are shown in table II.

Levels of morbidity, as judged by positive responses to the six dimensions of the Nottingham health profile in the whole sample of respondents are shown in table III. Also shown, for comparison, are the results of Curtis's study.

The results of the multiple regression modelling are shown in table IV: the model is built to predict a score of above or below the median on each dimension (separately) of the Nottingham health profile. The addition of the Jarman score to the model made no significant contribution to the regression for any of the six dimensions of the health profile, once age and sex had been included. Inclusion of a dummy variable for town (Colchester and Clacton) made no difference to the results, except that town was a significant predictor for energy (coefficient estimate 1.098, $\mathrm{SE}=0.013, \mathrm{p}=0.01$ ). Thus if one knows the mean age and sex of respondents in an enumeration district, knowing also the Jarman score of their district does not give any further help in predicting whether the response on any of the six Nottingham health profile dimensions will be above or below the median. The same was true if the Townsend score was used instead of the Jarman score. The most important predictor variable was age (significant for energy, pain, sleep, and mobility, but not emotions or social isolation); this is to be expected from published age and sex specific mean scores for the Nottingham health profile. ${ }^{8}$ The implication of, for example, the coefficient for age in predicting physical mobility (estimate 0.0834 ) is that for a given enumeration district, an increase of one year in mean age of the survey respondents of that district increases its odds of being in the top half of the distribution of mean mobility scores by a 
factor of $\exp (0.08)$, that is by a factor of 1.09 or $9 \%$. To set this in context, the median score for mobility in this sample of enumeration districts was 4.48 ; a positive response to the Nottingham health profile item "I find it hard to reach for things" attracts a score of $\mathbf{9 . 3}$ on the mobility scale.

\section{Discussion}

The purpose of this study was to see if Curtis's findings ${ }^{7}$ could be replicated in North East Essex. Using data from three areas of London (Tower Hamlets, Victoria, and Redbridge), she found that the Jarman score contributed significantly to the prediction of response to the Nottingham health profile, and concluded that the Jarman index might provide an useful surrogate indicator to estimate morbidity. This finding was not replicated in the present study of data from North East Essex. The same measures of morbidity (the Nottingham health profile) and deprivation (Jarman score assigned by enumeration district) were used in the two studies, and similar statistical analysis (multiple logistic regression inclusive of age and sex).

There are three possible explanations for the differing results of the two studies. Firstly, the methodology, and in particular the measure of deprivation, may be valid in London but not in Essex. Alternatively there may be a genuine difference between cities and towns in the effect that deprivation has on subjective health. Finally the difference may simply be due to chance: a true relationship not detected in this sample, or a null relationship falsely rejected on the evidence of Curtis's sample.

Let us consider first the methodological questions, which relate mostly to the use of deprivation scores. Curtis was careful to note that the Jarman score was "most likely to be effective in areas where sociodemographic profiles of the local population are highly contrasting"'. This raises the question of whether the present study looked at a more homogeneous population with little contrast in sociodemographic variables. I calculated Jarman scores relative to all North East Essex enumeration districts, and these scores cannot be compared directly with the scores in Curtis's paper (based on all enumeration districts in Greater London). The extent of contrast between enumeration districts in my study can be judged from the indicators which go to make up the Jarman score, and as noted the interquartile range was, for example, from $1.6 \%$ to $11.8 \%$ of elderly living alone and from $12.0 \%$ to $1.7 \%$ of overcrowded households, though with lesser variation in other elements of the score.

The Jarman score was developed ${ }^{7}$ from a questionnaire survey of a sample of general medical practitioners in London, and was designed to examine workload, not morbidity. The theoretical basis of the score, the uses to which it has been put, and its relevance outside London, have been heavily criticised. ${ }^{1-5}$ It could be argued that the present study merely adds to this weight of criticism by demonstrating that the Jarman score is able to predict morbidity in London but not in North East Essex. On the other hand Townsend's score performed no better as a predictor of response to five of the six Nottingham health profile dimensions, even though Townsend constructed his score specifically to overcome the theoretical weakness and London bias of Jarman's index. ${ }^{5}$ Furthermore Jarman and Townsend scores do correlate, in ecological studies at health authority and electoral ward level, with measures of health in areas other than London. ${ }^{510}$ On balance therefore I do not think that the negative finding of the present study is due to the use of measures of deprivation which are valid only in London, though this does remain a possibility.

There are some methodological problems with using the enumeration district as the unit for the deprivation score. Neither Jarman nor Townsend intended their scores for use at enumeration district level, where numerators for each component of the score are typically no more than 10-20 people. The accuracy of matching from postcode to enumeration district has also been doubted. ${ }^{11}$ Small numerators and inaccurate matching of post code to enumeration district would tend to obscure any relationship with enumeration district based scoring systems, and thus might account for the negative results of the present study. On the other hand the deprivation score of some larger unit (such as the electoral ward) may not characterise an individual's social environment very accurately if there are great contrasts within the ward. Furthermore any problems arising from the use of enumeration districts should be common to both studies and are thus unlikely to account for the difference in results.

Any census based measure becomes increasingly inaccurate or invalid with the passage of time, particularly if the character of a neighbourhood changes dramatically through redevelopment or "gentrification". The fieldwork for the Curtis study took place in 1982 and 1984; and for the present study in 1988. Although there has been a steady increase in population in Colchester and Clacton since 1981, the character of the 10 electoral wards from which the respondents were drawn has not changed substantially, so that their relative ranking - and thus Jarman score-may not have changed much between 1981 and 1988. It is however impossible to be certain of this point, and clearly Curtis's study will have characterised more accurately neighbourhood conditions at the time of interview for morbidity.

There are, then, several problems associated with the measurement of deprivation, and it is a matter of judgement whether or not they account for the difference between Curtis's findings and mine. What about the measurement of morbidity? The Nottingham health profile has come to be regarded as too insensitive to minor degrees of morbidity, even though it was developed for use in general population surveys, and it remains the best validated and most widely used instrument for general assessment of self rated health in United Kingdom populations. The Nottingham health profile was adequately sensitive for Curtis's study, and overall levels of morbidity as judged by this instrument were similar in the two studies, so that comparability between the two studies in measurement of morbidity should be good. Curtis's data were obtained entirely by household interview, and those in the present 
study by a combination of postal response and household interview, but the Nottingham health profile is designed for administration by either mode and there appears to be no systematic bias between different modes (EG Jessop, unpublished observations).

If we accept that the difference between the two studies is one of substance and not merely one of validity, what are the implications? The relationship between deprivation and health may genuinely be different in towns and cities. There is not space to explore this theme fully here, but if, as seems almost self evident, city life is different from life in a small town, it would not be surprising if the components of well being were also different in the two settings. The nexus between the social environment and an individual's health is his or her psychological wellbeing; and if deprivation is associated with morbidity in the city it may be simply that city dwellers are less resilient in the face of adversity. Such concepts are nebulous and difficult to study quantitatively, but they may be what gives rise to geographical differences in the relationship between deprivation (as we measure it) and health.

Either way Curtis's conclusion that the Jarman score may provide a useful surrogate indicator to estimate morbidity cannot be generalised; further work is required to define the geographical and social settings for which this conclusion is true. This is bad news for departments of public health in the National Health Service which, because of their responsibilities, need information on morbidity (not included in the census) about a complete resident population (which only a census provides).

More generally, this study adds further weight to the arguments against using census based deprivation scores as a proxy for health need in resource allocation formulas.

I am grateful to a great many people for help with this study, in particular Mr Ben Lavender for help with the mailing; Mrs Margaret Jones for excellent management of the household interviews; Mrs Mandy Bines for the data processing; and $\mathrm{Mr}$ Terence Bates for extracting the census data and computing the Jarman and Townsend scores; and Dr Paula Whitty for helpful comments on the manuscripts. Dr S E Curtis kindly provided unpublished data.

1 Smith GD. Second thoughts on the Jarman index. $B M F$ 1991; 302: 359-60.

2 Talbot $R$. Underprivileged areas and health care planning: implications of use of Jarman indicators of urban deprivation. BMF 1991; 302: 383-6.

3 Carr-Hill RA, Sheldon T. Designing a deprivation payment for general practitioners: the UPA $(8)$ wonderland. $B M F$ for general practition

4 Carstairs V, Morris R. Deprivation: explaining differences in mortality between Scotland and England and Wales. in mortality between BMF 1989; 299: 886-9.

5 Townsend P, Phillimore P, Beattie A. Health and deprivation. London: Croom Helm, 1988.

6 Leavey R, Wilkin D. A comparison of two survey measures of health status. Soc Sci Med 1988; 27: 269-75.

7 Curtis SE. Use of survey data and small area statistics to assess the link between individual morbidity and neighbourhood deprivation. $f$ Epidemiol Community Health 1990; 44: 62-8.

8 Hunt SM, McEwen J, McKenna SP. Measuring health status. London: Croom Helm, 1986.

9 Jarman B. Identification of underprivileged areas. BMF 1983; 286; 1705-9.

10 Mays N, Chinn S. Relation between all cause standardised mortality ratios and two indices of deprivation at regional and district level in England. $\mathcal{f}$ Epidemiol Community and district level in Health 1989; 43: 191-9.

11 Senior ML. The Jarman index. BMf 1991; 302: 661 (letter). 